\title{
Complex landscape topography can facilitate local adaptation during a range shift
}

\author{
Robert Fitt ${ }^{1}$ and L. T. Lancaster ${ }^{2}$ \\ ${ }^{1}$ University of Liverpool \\ ${ }^{2}$ University of Aberdeen
}

May 6, 2021

\begin{abstract}
Warming climates provide many species the opportunity to colonise newly-suitable regions at higher latitudes and elevations. Despite becoming warmer, higher latitudes and elevations nevertheless offer novel climatic challenges, such as greater thermal variability and altered frequency of weather events, and these challenges exert selection on expanding populations. However, high gene flow and genetic drift during the expansion phase may limit the degree to which species can adapt to novel climatic conditions at the range front. Here we examine how landscape topographic complexity influences the opportunity for local adaptation to novel conditions during a range shift. Using RAD-seq data, we investigated whether elevation, latitude, climatic niche differentiation, and gene flow across a complex landscape were associated with signatures of adaptation during recent range expansion of the damselfly Ischnura elegans in Northeast Scotland. Our data revealed two distinct routes of colonisation, with admixture between these routes resulting in increased heterozygosity and population density. Expansion rates, assessed as directional rates of gene flow, were greater between more climatically similar sites than between climatically divergent sites. Significant genetic structure and allelic turnover was found to emerge near the range front at sites characterised by high elevation, low directional gene flow, and high spatial differentiation in climate regimes. This predictive combination of factors suggests that landscape complexity may be a prerequisite for promoting differentiation of populations, and providing opportunities for local adaptation, during rapid or contemporary range shifts
\end{abstract}

\section{Hosted file}

FittLancasterGenomics_E\&E.pdf available at https://authorea.com/users/412287/articles/521021complex-landscape-topography-can-facilitate-local-adaptation-during-a-range-shift 